\title{
Simulation-Based Analysis on Novel Vacuum Actuation Concept for Soft Robotic Finger Development
}

\author{
Noer Fadzri Perdana Dinata ${ }^{1}$ Hasan Basri ${ }^{1, *}$ \\ ${ }^{1}$ Mechanical Engineering Master Study Program, Universitas Sriwijaya, Jalan Srijaya Negara Bukit Besar \\ Palembang, Sumatera Selatan, 30139, Indonesia \\ *Corresponding author.Email: hasan_basri@unsri.ac.id
}

\begin{abstract}
Grasping of fragile objects, such agricultural products, is a currently active area of research which is advancing continuously throughout the years. Although proven versatility has been achieved through conventional robotic gripper, the recent favourable option is to utilized soft robotic grippers to hold various shapes and interacting with unstructured environments. The principal concept of soft grippers is to be able to handle or manipulate various fragile items without harming the object. This study introduced a structured printable soft vacuum actuator that is covered by a commercially available latex balloon as the membrane. These actuators are directly manufactured into segmented soft robotic fingers using commercially affordable fused deposition modelling (FDM) 3D printer. Due to its flexible structural deformation, the task could be achieved without additional complication like their rigid-bodied counterparts. In this study, the behaviour of these actuators is predicted using numerical analysis in finite element modelling. Furthermore, several structures have been investigated in order to observe any relation between the stress distributions on the material surface, to the actuator shape. Some advantages acquire from this development are its economical production cost, vastly available material, straight-forward fabrication, and large payload to weight ratio. While the gripper only weighs approximately 63 grams, is capable of lifting to 800 grams load. It is clearly shown from the simulated result; those specific configurations could give an optimized design of up to $50 \%$ for motion and repetitive actuation.
\end{abstract}

Keywords: Vacuum actuator, soft robotic, soft gripper, $3 d$ printed soft actuator

\section{INTRODUCTION}

Simple tasks in human perspective is exasperatingly tricky for robots, especially when it comes to dealing with fragile objects [1]-[4]. In soft robotics field, this task is fulfilled by replacing the concept of finger articulated motion to material deformation. The notion of this approach is to shape the soft gripper around the object upon contact. At present, excessive usage of positive pneumatic pressure as the actuation method in soft robotic developments have been reported a number of times [5]-[8]. In this study, we introduce a vacuumpowered soft robotic finger, where the mechanism depends on the flexible $3 \mathrm{D}$ printed structure. To complete the fabrication, the structure is subsequently put inside a membrane that acts as a vacuum chamber.
To demonstrate the capability of the fabricated fingers to grasp objects, a three-finger configuration was developed. Furthermore, the three-finger gripper was tested to grasps and picks up various types of fruits. These soft grippers are perfectly applicable in agricultural field where picking and moving fruits or vegetables is required. Since the actuators are capable to adapt to the object geometry, no force sensor is necessary as the feedback. The demonstrated used of this vacuum actuated gripper is presented in Figure 1

In recent soft robotic study [9], [10], the concept of vacuum actuated soft finger has taking a lot of attention from the researcher. This is particularly correct considering its fail-safe operation, by limiting the material strain based on its geometric boundary [10]. Contrary to the positive pressured actuation method, the vacuum actuated finger motion is limited based on the 
geometry or the structure contact, thus limiting the strain applied to the material.
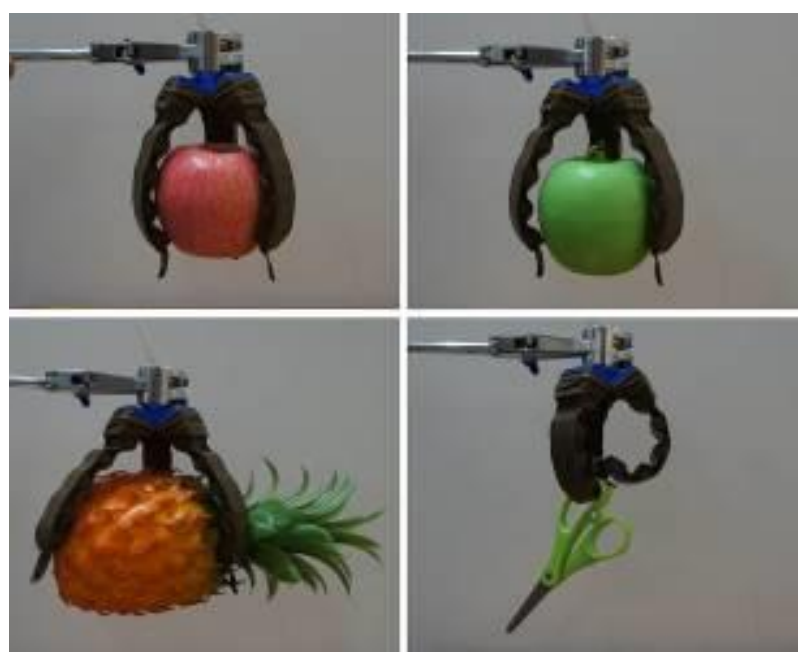

Figure 1 The prototype of the soft vacuum finger was configured as a three-finger gripper, and further put under vacuum pressure condition to actuate the gripping state.

This study aim to develop a novel soft actuated finger and characterize its behaviour. The characterization was done by considering the structure as one actuated soft finger. The modelling and finite element analysis was utilized to predict the trajectory of motion of the finger. Furthermore, the stress intensity factor that are related to performance characterization is also being measured in this study.

\section{MATERIALS AND METHOD}

\subsection{Design and Actuation Concept}

The actuators have been designed in Solidwork 2019 (Dassault System, USA). In order to keep the system from folding in the in-plane direction, the actuators were built with multiple vacuum chambers that separated by a triangular rigid body. Furthermore, the actuators were modelled with a strain limiting layer thickness twice the size of the upper shell. The dimension and design of the finger structure is presented in Figure 2. Following structure design was used to acquire the deviation measurement on the 3D printed soft finger structure. The dimension given was copied from the report prepared by Charbel Tawk, et al [11]. However, some modification was done to the reported design to simplify the $3 \mathrm{D}$ printing process.

The actuation concept were prepared as presented in Figure 2c. To applied vacuum on the printed structure, each samples were covered in a flexible membrane. The finger is actuated due the applied pressure on the structure. This is where the optimized dimension play its role especially on the actuation layer part. Therefore, by the time the vacuum pressure applied to the diagram, as shown in Figure $4 c$, the finger structure will start to bend.

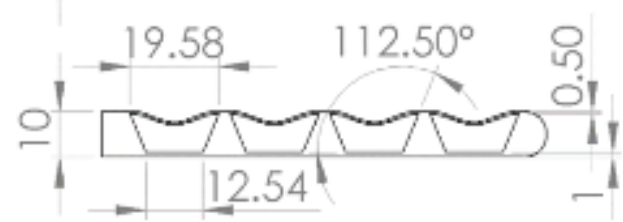

(a)

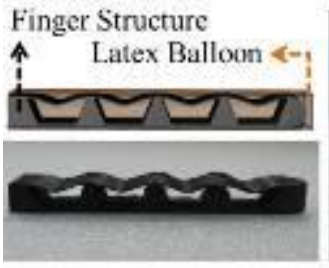

(b)

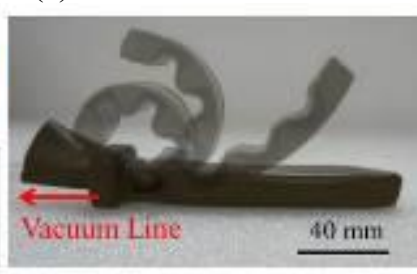

(c)
Figure 2 (a) Dimension of one finger structure, the fingertip and the base point are designed in such a way, for easy insertion of vacuum tube and latex balloon. (b) 3D printed structure, to apply vacuum, the structure will then be put inside balloon. (c) Finished product; the membrane could be chosen arbitrarily, latex balloon is used for its convenient to acquire.

\subsection{Trajectory and Stress Intensity Factor Analysis}

Finite element analysis was performed in Solidworks Simulation feature. A Static Non-linear Analysis was performed to simulate the deformation of one segment module, as shown in Figure 3, in order to minimize computational resources.

The proposed 3D printed material used, which is thermoplastic polyurethane (TPU), is considered as Hyperelastic Material. The parameter utilized in the modelling is Mooney-Rivlin 5 parameter models [10]. Parameter value was set in accordance to curve fitting result of the material tensile test. Higher order tetrahedral elements were utilized as the mesh to the model. Fixed geometry was set to the base of the actuator as boundary conditions. Negative pressure applied to the outer shells of the structure. The $\mathrm{X}$ and $\mathrm{Y}$ displacement shown in Fig. 1(c), will then be calculated further mathematically, using Matlab, by estimating the trajectory using forward kinematic analysis [11]. Therefore, the positioning on one finger structure could be express as a Homogenous Transformation matrix shown in equation (1).

$$
\left[\begin{array}{c}
A_{x} \\
A_{y} \\
A_{z} \\
1
\end{array}\right]=\left[\begin{array}{cccc}
r_{11} & r_{12} & r_{13} & A_{x B} \\
r_{21} & r_{22} & r_{2 a} & A_{y B} \\
r_{21} & r_{22} & r_{23} & A_{z B} \\
0 & 0 & 0 & 1
\end{array}\right]
$$



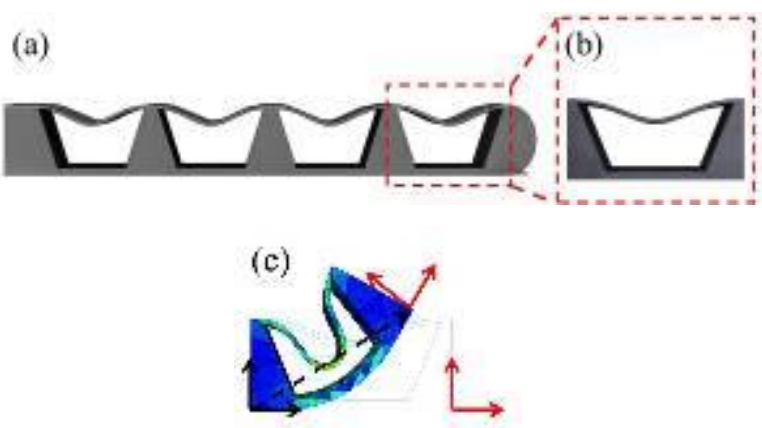

Figure 3 (a) Analysis on one finger structure is heavy on resources. (b) Therefore, one segment is simulated under evenly distributed $-60 \mathrm{kPa}$ pressure (c) Simulated deformation; the $\mathrm{X}$ and $\mathrm{Y}$ displacement on this simulation will then be brought up for further mathematical analysis.

Where, $\left[\begin{array}{lll}P_{11} & -m & T_{a a}\end{array}\right]$ is a rotation matrix, and the vector $\left[A_{B}\right]$ is the displacement vector from the reference origin. Furthermore, the fabricated finger was undergoing an experiment to verify the trajectory analysis from the simulation result. The experiment was done by using a video labeler analysis from Matlab (R2019a; The MathWorks, Inc., Natick, MA). The toolbox was utilized to follow the red dot movement under vacuum actuated condition.

\section{RESULTS AND DISCUSSION}

\subsection{Trajectory Analysis}

Actuator motion prediction was obtained from the finite element simulation. The actual trajectory of the printed structure was predicted by the simulations, as presented in Figure 4. The graph shown the trajectory of each segment of the modelled finger. Taking note from the simulation, the 3D modelled geometry was simulated where the latex membrane covering the internal structure were ignored. Through the results of the pressure distribution on the structure, the correlation between bending angle and stress on the actuation layer can be estimated. The purpose of this analysis is to approximate energy stored in the TPU material under load. Hence, its relation to performance deviation can be studied.

Quantitative comparison for the deformations are calculated based on four corresponding segment marked on the triangular rigid body. This trajectory result indicates that the parameter generation using FEM analysis is adequate to further examined as variable to stress intensity factor, which will be discuss on the next section.

\subsection{Stress Intensity Factor}

The previous trajectory observation may support the hypothesis that the upper layer is taking the main role for

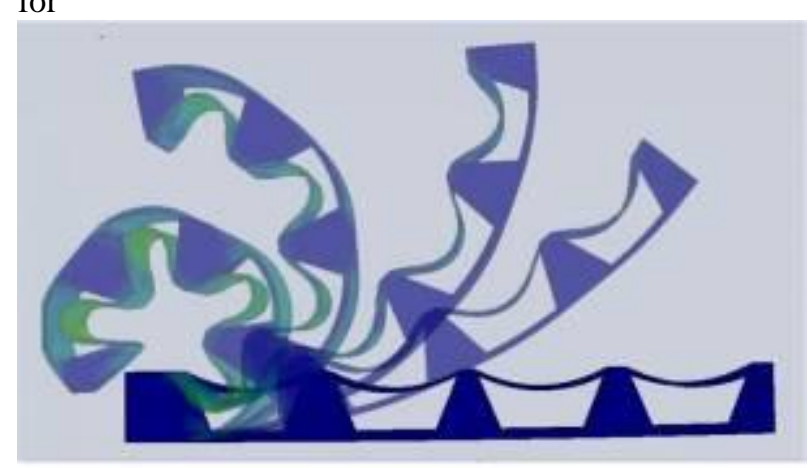

(a)

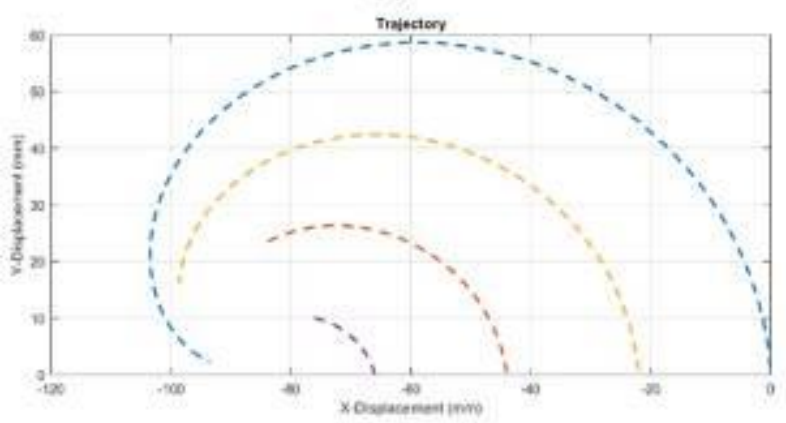

(b)

Figure 4 (a) Simulated motion using Finite Element Analysis software. (b) Trajectory result based on the given motion from Figure 3(a).

the bending actuation. Hence, the examination on the stress intensity factor on the upper layer only, is adequate to measure the performance under repetitive actuation. To put emphasize on the geometry, 3 different geometry was considered as the actuation parameter. The result of 3 distinguished geometry configuration is shown in Figure 5, below.

The result shown is in accordance with series of compressive tests with large pressure, proof of cyclic softening and Mullin's effect which had examined intensively [12]. Further research [13] is based on the characterization of TPU stress-strain behaviour by taking both mechanical and thermal variables into consideration when a cyclic stress is imposed on the material.. The hyperelastic material stress-strain behaviour can be simply represented by defining a dynamic elastic modulus $E_{d}$, at different temperatures, frequencies, and imposed strains, according to classic linear viscoelasticity,.

$$
E_{d}=\frac{\sigma_{a x}}{\varepsilon_{a}}
$$

where $\sigma_{\bar{\alpha}}$ and $\varepsilon_{\tilde{\sigma}}$ are the alternate nominal strain and stress components in a loading cycle, respectively. 
The result shown also support Avanzini et al, that $E_{d}$ values decline as the applied strain rises at all levels within the considered strain range. Increased temperature

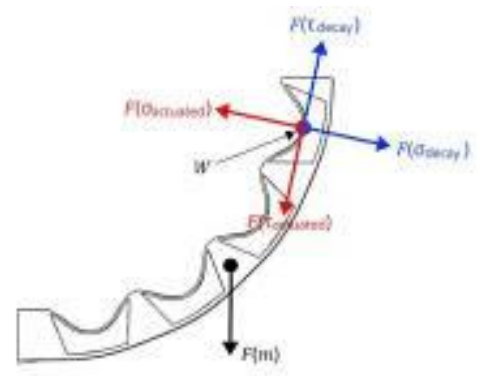

(a)
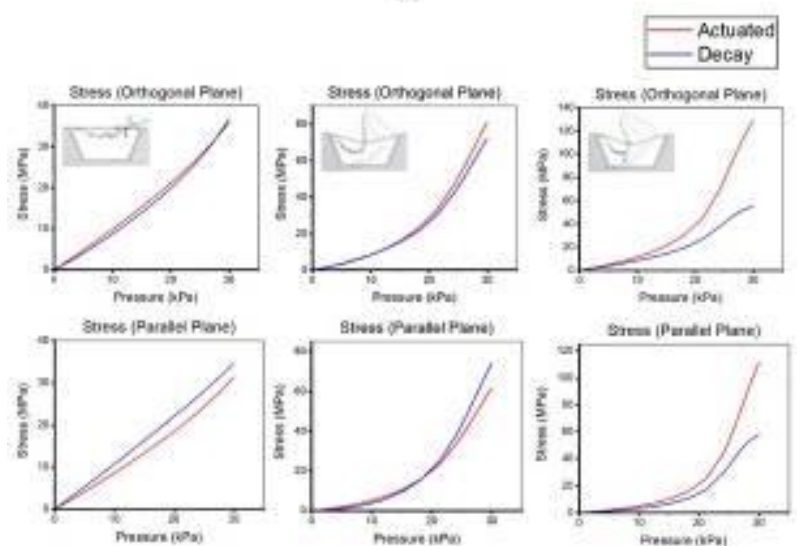

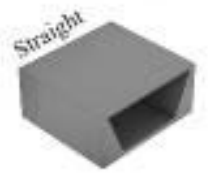

(b)

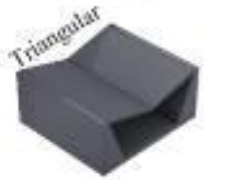

(c)

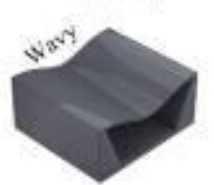

(d)
Figure 5 (a) Diagram of stored energy within actuation, (b) Stress distribution on the straight upper layer configuration, (c) Stress distribution on the triangular upper layer configuration, (d) Stress distribution on the wavy upper layer configuration.

values contribute to further reduction. Therefore, the softening activity that leads to $E_{d}$ reduction is indeed strain-dependent. Particularly with increasing strain, the amount of softening increases. It may be beneficial to note the close relation in the sense of viscoelasticity theories, between dynamic elastic modulus $E_{d}$ to the storage module $E$, as defined in Equation 3,

$$
E^{y}=\frac{\sigma_{a x}}{\varepsilon_{a}} \cos \delta
$$

Furthermore, Omar et al.[14] had introduced the mathematical formulation and analysis of the fatigue values and the cycle number, to calculate the wear and crack growth behaviour on a polymer. The correlation of several thermoplastics were investigated in a dry or wet state. The Paris-Erdogan law used in equation 4 sets the correlation between the crack growth rate and the stress intensity factor. Omar's idea was to find the relation between the wear rate and the cyclic load repetition until failure, as shown in equation 5. This is where the variables used in this study will be significant in term of performance repeatibility. Several stress intensity factor based on the geometry, as presented in Figure 5 will be the driving factor to affect the stress intensity factor $K$.

$$
\begin{gathered}
\frac{d a}{d N}=A \cdot K^{n} \\
W_{F} \propto \frac{1}{N_{f}} \propto A \cdot \Delta \sigma^{n} \cdot \sqrt{\pi^{n}} \cdot a_{0}^{\frac{1 n}{2}-1} \cdot\left(\frac{n}{2}-1\right)
\end{gathered}
$$

where $a$ is the crack length, $N$ is the repetitive cyclic load value, $A$ is the material constant, $K$ is the stress intensity factor, $n$ is slope of the straight, $W_{F}$ is the wear rate, and $\Delta \sigma^{n}$ is the difference in applied stress. An assumption was made for the value of $a$ and $a_{0}$. Considering the finger structure, the value of the crack length was assumed happened along the width of the structure, therefore could be set at $20 \mathrm{~mm}$. This is where the data from FEM Analysis utilized, to measure the performance of the actuator for fatigue condition, which will be considered as a future works.

\section{CONCLUSIONS}

Compared with conventional positive pressured soft pneumatic actuators, the vacuum actuated soft finger have a distinctive advantage. Straightforward production, in which the actuators can be easily produced using FDM 3D printers that are commercially available. The trajectory from the simulation shown a relatively accurate prediction of the finger motion. The verification of the simulation will be considered as a future works.

This study has shown that the soft actuator can be analysed using finite element method. The result of the simulated stress intensity factor has shown that by configuring the upper layer to approximately wavy structure, the design could be optimized up to $50 \%$ compare to other geometry. The application of this development is not restricted exclusively to grasping robot but also for a wide variety of robotic applications, including locomotive robots, as well as artificial muscles., which also be considered for the future works.

\section{ACKNOWLEDGMENTS}

We gratefully thank the Mechanical Engineering Masters Study Program, Faculty of Engineering, Universitas Sriwijaya for their strong support of this study, along with all Faculty members for their 
encouragement and many fruitful discussions on this research.

\section{REFERENCES}

[1] A. Bicchi, "Hands for dexterous manipulation and robust grasping: A difficult road toward simplicity," IEEE Trans. Robot. Autom., vol. 16, no. 6 , pp. 652-662, 2000, doi: $10.1109 / 70.897777$.

[2] J. J. Craig, Introduction to Robotics: Mechanics and Control, vol. 41, no. 4. 2004.

[3] D. T. Pham and S. H. Yeo, "Strategies for gripper design and selection in robotic assembly," Int. J. Prod. Res., vol. 29, no. 2, pp. 303-316, 1991, doi: 10.1080/00207549108930072.

[4] B. Siciliano and O. Khatib, Eds., Handbook of Robotics. Springer US, 2008.

[5] J. Hughes, U. Culha, F. Giardina, F. Guenther, A. Rosendo, and F. Iida, "Soft Manipulators and Grippers: A Review," Front. Robot. AI. Front. Media S.A., vol. 3, no. November, pp. 1-12, 2016, doi: 10.3389/frobt.2016.00069.

[6] R. Shepherd et al., "Multigait soft robot," in Proceeding of the National Academy of Sciences of the United Stated of America, 2011, pp. 20400-20403, doi: https://dx.doi.org/10.1073/pnas.1116564108.

[7] A. Miriyev, K. Stack, and H. Lipson, "Soft material for soft actuators," Nat. Commun., vol. 8, no. 1, pp. 1-8, 2017, doi: 10.1038/s41467017-00685-3.
[8] N. Vasios, A. J. Gross, S. Soifer, J. T. B. Overvelde, and K. Bertoldi, "Harnessing Viscous Flow to Simplify the Actuation of Fluidic Soft Robots," vol. 00, no. 00, pp. 1-9, 2019, doi: 10.1089/soro.2018.0149.

[9] M. A. Robertson and J. Paik, "New soft robots really suck : Vacuum-powered systems empower diverse capabilities," Sci. Robot., vol. 2, no. 9, pp. 1-12, 2017, doi: https://dx.doi.org/10.1126/scirobotics.aan6357.

[10] C. Tawk, G. M. Spinks, and G. Alici, "3D Printable Linear Soft Vacuum Actuators : Their Modeling , Performance Quantification and Application in Soft Robotic Systems," IEEE/ASME Trans. Mechatronics, vol. 24, no. 5, pp. 2118-2129, 2019.

[11] C. Tawk et al., "Bioinspired 3D Printable Soft Vacuum Actuators for Locomotion Robots, Grippers and Artificial Muscles," Soft Robot., vol. 5, no. 6, pp. 685-694, 2018, doi: 10.1089 /soro.2018.0021.

[12] H. J. Qi and M. C. Boyce, "Stress-strain behavior of thermoplastic polyurethanes," Mech. Mater., vol. 37, no. 8, pp. 817-839, 2005, doi: 10.1016/j.mechmat.2004.08.001.

[13] A. Avanzini and D. Gallina, "Effect of cyclic strain on the mechanical behavior of a thermoplastic polyurethane," J. Eng. Mater. Technol. Trans. ASME, vol. 133, no. 2, pp. 1-9, 2011, doi: 10.1115/1.4003101.

[14] M. K. Omar, A. G. Atkins, and J. K. Lancaster, "The role of crack resistance parameters in polymer wear," J. Phys. D. Appl. Phys., vol. 19, no. 2, pp. 177-195, 1986, doi: 10.1088/0022$3727 / 19 / 2 / 007$. 